\title{
Diagnostics of Bearing Defects Using Vibration Signal
}

\author{
Kayode Oyeniyi Oyedoja
}

\begin{abstract}
Current trend toward industrial automation requires the replacement of supervision and monitoring roles traditionally performed by humans with artificial intelligent systems. This paper studies the use of hybrid intelligent system in Diagnosis of rotating machinery bearing defects. Vibration signals were collected for normal and various faulty conditions of the ball/roller bearing of the machinery. The acquired signal was processed with FFT and PSD in MATLAB to obtain the characteristic amplitudes from the frequency domain spectra of the signals. The obtained amplitude vector was used to train an adaptive neurofuzzy inference system (ANFIS) to classify and recognize normal and different faulty states. The system was tested, checked and validated with different sets of signal data. The validation data attests to the structural stability and performance of the system.
\end{abstract}

Index Terms-ANFIS, bearing fault, fault diagnosis, MATLAB, rotating machinery vibration,

\section{INTRODUCTION}

Bearing defect diagnosis is based on the facts that mechanical systems produce vibration while operating, and that important information about the state of operation of the system can be extracted from the analysis of the vibration signal. Generally, vibration levels are low and constant when a machine is operating properly; however, when faults begin to develop or when some of the dynamic processes in the machine change, the vibration levels also change [1], [2] In the frequency domain, changes in vibration levels translate into changes in the shape of the frequency

For many machines, the vibration frequency spectrum has characteristic shape when the machine is operating properly, and it has other features for different faults that may appear. Diagnosis of faults can be accomplished in many cases by detecting features in the frequency spectrum which are known to be related to particular faults. All vibration monitoring techniques are based fundamentally on the recording and quantification of small vibration impulses. Often, spectral features associated with specific defects can be generated at frequencies that can be calculated from formulae derived from models; however, the task of diagnosis is complicated by a series of factors, such as noise, presence of multiple faults, and severity of fault and speed changes. Fault diagnosis is also complicated by the fact that fundamental frequency components often disappear at advanced stages of the defect, while harmonic components remain [3], [4].

When performing vibration monitoring of rolling element

Manuscript received October 16, 2012; revised November 16, 2012.

K. O. Oyedoja is with the Department of Technical Education, Emmanuel Alayande College of Education, Oyo, Oyo State, Nigeria (email: dojakay@yahoo.com,) bearings, the emphasis is more on the content of the spectrum, than on its amplitude. Amplitudes of bearing characteristics frequencies often begin to decrease as condition worsens. Therefore more importance should be attributed to the fact that a multiple number of fault frequencies are appearing in the spectrum than to the exact amplitude. This fact led to incorporation of fuzzy logic into the classification system. The soft boundaries in fuzzy logic environments, obtained by membership functions, are of special interest because their use results in flexible, more human-like classifications. On the other hand, neural networks provide a viable technique for the analysis of vibration data because of their inherent ability to operate on noisy, incomplete or sparse data and to model processes from actual system data. By combining neural networks and fuzzy logic we are able to take advantage of the strengths of both approaches.

Most vibration analysis instruments today utilize a Fast Fourier Transform (FFT) which is a special case of the generalized Discrete Fourier Transform and converts the vibration signal from its time domain representation to its equivalent frequency domain representation. However, frequency analysis (sometimes called Spectral Analysis or Vibration Signature Analysis) is only one aspect of interpreting the information contained in a vibration signal. Frequency analysis tends to be most useful on machines that employ rolling element bearings and whose main failure modes tend to be the degradation of those bearings, which typically exhibit an increase in characteristic frequencies associated with the bearing geometries and constructions.

\section{FiVE BASIC FREQUENCIES RELATED TO ROLLING BEARING DYNAMICS.}

Roller bearings generally consist of two concentric rings with a set of rolling elements running in their tracks. These two rings are called the outer raceway and inner raceway, respectively. Standard shapes of rolling elements include the ball, cylindrical roller, tapered roller, needle roller, and symmetrical and unsymmetrical barrel roller, [5], [6], [7]. Usually, the rolling elements in a bearing are guided in a cage that ensures uniform spacing and prevents mutual contact.

There are five basic motions that can be used to describe the dynamics of a bearing movement and each motion generates a unique frequency response. These five frequencies, shown in Fig. 1, are defined as: shaft rotational frequency $\left(\mathrm{f}_{\mathrm{S}}\right)$, fundamental cage frequency $\left(f_{F C F}\right)$, ball pass outer raceway frequency $\left(f_{B P O F}\right)$, ball pass inner raceway frequency $\left(f_{B P I F}\right)$, and ball rotational frequency $\left(f_{B}\right)$.

In the following derivations, as shown in figure $1, f_{i} f_{c}$ and $f_{o}$ represent the rotating frequency of the inner raceway, ball 
center and outer raceway respectively. $D_{b}$ is the ball diameter, $D_{c}$ is the cage diameter of the bearing and is measured from a ball center to the opposite ball center, and $\theta$ is the contact angle of bearing.
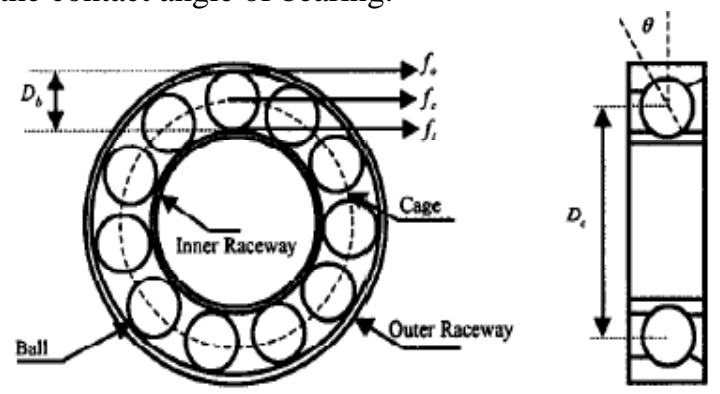

Fig. 1. The structure of a ball bearing [8].

\section{BEARING FAULT FREQUENCIES}

A bearing generates vibration when it gets defected due to steady manner of mechanical friction. Vibration causes strong harmonics and sidebands. Given the geometry of the bearing and the frequency of the running speed of the rotating machinery, fault frequencies could be distinguished from other frequency components [11]. For a bearing shown in Fig. 1 with the outer ring being stationary, bearing fault or key frequencies are as follows:

Fundamental Cage Frequency (f FCF)

$$
f_{F C F}=\frac{f_{s}}{2}\left(-\frac{D_{b}}{D_{c}} \cos \Theta\right) .
$$

Ball Pass Outer Raceway Frequency (f BPOF)

$$
\begin{gathered}
f_{B P O F}=N_{B}\left|f_{F C F}-f_{o}\right|, \\
=N_{B}\left|\frac{f_{S}}{2}\left(1-\frac{D_{b} \cos \theta}{D_{b}}\right)-0\right|, \\
=\frac{N_{B}}{2} f_{S}\left(1-\frac{D_{b} \cos \theta}{D_{c}}\right) .
\end{gathered}
$$

Ball Pass Inner Raceway Frequency (fBPIF)

$$
\begin{aligned}
& f_{B P I F}=N_{B}\left|f_{F C F}-f_{i}\right|, \\
= & N_{B}\left|\frac{f_{S}}{2}\left(1-\frac{D_{b} \cos \theta}{D_{c}}\right)-f_{S}\right|, \\
= & \frac{N_{B}}{2} f_{S}\left(1+\frac{D_{b} \cos \theta}{D_{c}}\right) .
\end{aligned}
$$

Ball Rotational Frequency (fB)

$$
f_{B}=\frac{D_{c}}{2 D_{b}} f_{S}\left(1-\frac{D_{b}^{2} \cos ^{2} \theta}{D_{c}^{2}}\right) \text {. }
$$

\section{BEARING VIBRATION CHARACTERISTICS}

All frequencies present in the spectrum of a bearing vibration must have been generated by some source. In practice, these sources exist in the form of some inherent defect in, or looseness of, the bearings, [10], [11].

For defects on the raceway of a rolling bearing, each time a roller hits the defective raceway, the: corresponding ball pass inner raceway frequency, $f_{B P I F}$ or ball pass outer raceway frequency, $f_{B P O F}$ will be generated. If the defective area is large, harmonics of $f_{B P I F}$, or $f_{B P O F}$ will also be present. The existence of these harmonics can help to indicate the severity of the defect.

The roller hits both the inner raceway and outer raceway each time it spins on its own axis. Therefore, for defects on the roller, twice the ball rotational frequency $\left(2 f_{B}\right)$ and the fundamental cage frequency $\left(f_{F C F}\right)$ will usually be generated. In most cases, these frequencies will be modulated with other existing frequencies and result in a more complicated spectrum. The simplest spectrum for bearing vibration is the spectrum caused by bearing looseness. Only fundamental cage frequency $\left(f_{F C F}\right)$ or shaft rotational frequency $\left(f_{S}\right)$ and their harmonics exist for this case. Table I lists the bearing vibration frequency characteristics for various faults.

TABLE I: VIBRATION FREQUENCY CHARACTERISTICS

\begin{tabular}{|l|l|l|}
\hline \multicolumn{1}{|c|}{$\begin{array}{c}\text { Location of } \\
\text { defects }\end{array}$} & \multicolumn{1}{|c|}{ Frequency } & \multicolumn{1}{c|}{ Observations } \\
\hline $\begin{array}{l}\text { Bearing } \\
\text { looseness }\end{array}$ & $f_{S}, f_{F C F}$ & Modulated by $2 f_{B}, f_{F C F}$ \\
\hline $\begin{array}{l}\text { Rolling } \\
\text { elements }\end{array}$ & $f_{B P I F}, f_{B P O F}$ & $\begin{array}{l}\text { An increase in the severity of } \\
\text { the defects results in higher } \\
\text { order }\end{array}$ \\
\hline Inner raceway & $f_{B P I F}$ & harmonics being produced \\
\hline Outer raceway & $f_{B P O F}$ & \\
\hline
\end{tabular}

\begin{tabular}{|c|c|c|c|}
\hline \multirow[b]{2}{*}{ Fault state } & \multicolumn{3}{|c|}{ Fault type } \\
\hline & $\begin{array}{c}\text { Ball pass outer } \\
\text { raceway }\end{array}$ & Ball spin & $\begin{array}{c}\text { Ball pass inner } \\
\text { raceway }\end{array}$ \\
\hline 0 (normal) & 0 & 0 & 0 \\
\hline 1 & 0 & 0 & 1 \\
\hline 2 & 0 & 1 & 0 \\
\hline 3 & 0 & 1 & 1 \\
\hline 4 & 1 & 0 & 0 \\
\hline 5 & 1 & 0 & 1 \\
\hline 6 & 1 & 1 & 0 \\
\hline 7 & 1 & 1 & 1 \\
\hline
\end{tabular}

\section{A. Definition of Normal and Different Faulty Conditions}

For our purpose, we need to define normal and faulty operating conditions of the bearing in a digital form that can be used for computational purposes. This is done by representing the presence or absence of a particular fault type with bit sets as stated in Table II.

TABLE II: DEFINITION OF NORMAL AND DIFFERENT FAULTY CONDITIONS

1 indicates the presence of particular type of fault in a certain fault state

0 indicates otherwise

\section{EXTRACTION OF BEARING VIBRATION CHARACTERISTICS}

The experimental setup is used to acquire vibration signals with varying severity for each of seven different bearing defects. These are the vibrations caused by bearing looseness, by defects on the inner raceway, by defects on the rolling element, and combinations of the above. Each signal consists of 2048 sampling points with a sampling time of 1 second. An FFT is performed to extract the main characteristics of these vibration signals. This procedure is shown in this equation, 


$$
D(K)=\sum_{n=0}^{N-1} d(n) e^{-\frac{f 2 \pi k n}{N}}, K=0,1, \ldots \ldots, N-1
$$

where $\mathrm{N}=2048$ and $d(n), 0 \leq n \leq \mathrm{N}-1$ denotes the time domain data. The power spectrum of the vibration is obtained as follows:

$$
\begin{aligned}
& P(0)=|D(0)|^{2}, \\
& P(k)=\left[|D(k)|^{2}+|D(N-k)|^{2}\right] \quad k=1,2, \ldots \ldots \frac{N}{2}-1, \\
& P\left(\frac{N}{2}\right)=\left|D\left(\frac{N}{2}\right)\right|^{2} .
\end{aligned}
$$

Next, we construct basic frequency amplitude vectors to represent different bearing vibrations. These vectors are created from the power spectrum of the vibration signal and consist of the five basic frequencies; with varying amplitudes based on the defect present. Since the spectral components near the five basic frequencies are also important, when generating the vector we consider a frequency band of $10 \mathrm{~Hz}$ for each basic frequency. That is,

frequency band $=[f-5, f+5]$

where $f$ is the basic frequency.

For the five different basic frequencies, the basic frequency amplitude vector and its elements can be represented as:

$$
F(f)=\sum_{f_{s}=f-5}^{f+5} P\left(\frac{f_{k}}{\Delta f}\right), \quad \Delta f=\frac{1}{T_{s}},
$$

where $\boldsymbol{T}_{\boldsymbol{s}}$ is the sampling time.

Therefore, the five basic frequencies are:

$$
\begin{aligned}
& x_{f_{S}}=F\left(f_{s}\right), \\
& x_{f_{F C F}}=F\left(f_{F C F}\right), \\
& x_{f_{B P O F}}=F\left(f_{B P O F}\right), \\
& x_{f_{B P I F}}=F\left(f_{B P I F}\right), \\
& x_{f_{B}}=F\left(f_{B}\right) .
\end{aligned}
$$

The basic frequencies amplitude vector is given by

$$
X=\left[\frac{x_{f_{S}}}{P}, \frac{x_{f_{f c f}}}{P}, \frac{x_{f_{B P O F}}}{P}, \frac{x_{f_{B P I F}}}{P}, \frac{x_{f_{B}}}{P}\right],
$$

where

$$
P=\sum_{K=0}^{N-1} P(K)
$$

Amplitudes of these frequencies vary according to type of defect that may occur in the bearing.

Table I shows that the vibration generated by defects in the bearing will show one, or some combination of several, of the five basic frequencies. Many different literatures, [3], [8] have discussed the usage of these five basic frequencies to identify defects in a bearing. In the normal machine fault detection process, the expert will survey the time-domain signal and the frequency spectra of bearing vibration to determine if there are any defects within the bearing.
However, a problem with this approach is that the experience, which is gained over a period of many years, is a very expensive and inefficient use of resources.

Therefore, if we can train artificial neurofuzzy network to learn the knowledge of the vibration expert, we can reduce the cost of fault detection and improve the efficiency.

Faults in a ball/roller bearing may occur on the inner raceway, the ball or roller itself or on the outer raceway. The presence of fault on any of this element or in combination is indicated in the signal by marked increase in the amplitude at the characteristic frequency for such element. This is the reason why we transform our data from time-based sample to frequency based with FFT and the calculation of power spectra density. The power spectra density clearly shows the marked increase in amplitude at the characteristic frequency for a particular fault type.

The Fig. 2 shows the time domain and spectra plot of samples of the acquired data, one data sample first for normal and then for each of the seven fault states.

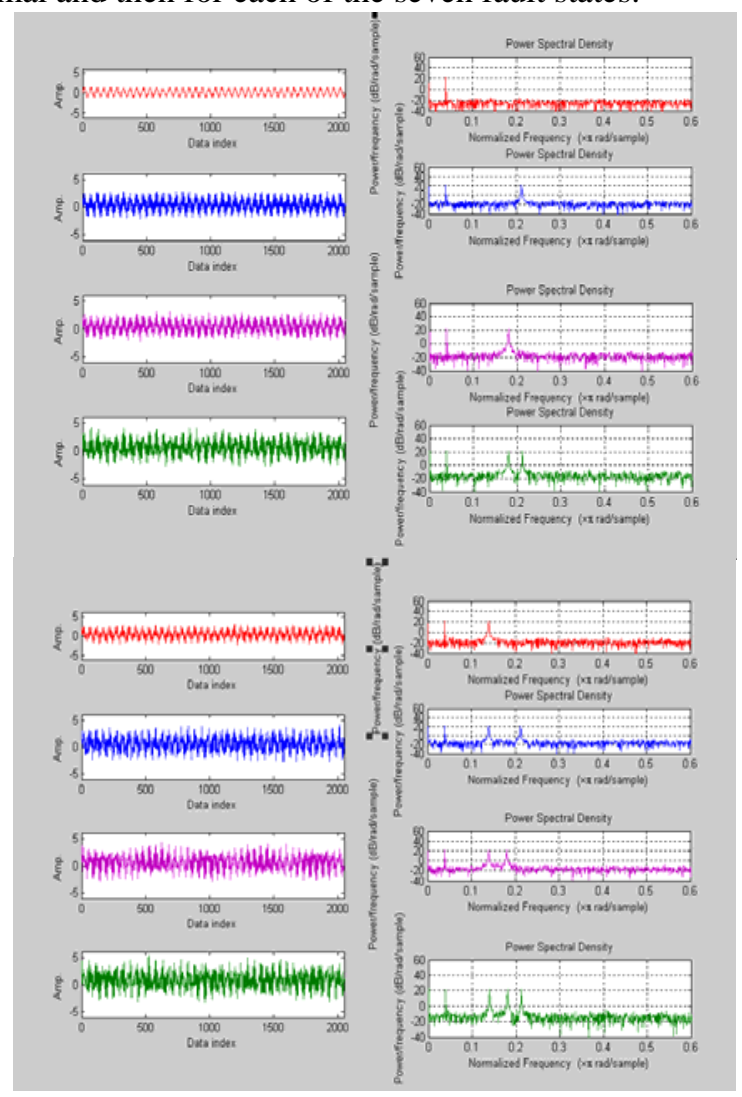

Fig. 2. Time domain and frequency spectra plot of the vibration signal samples

\section{A. ANFIS for Bearing Fault Detection}

Sugeno type adaptive neuro fuzzy inference system with four input nodes and one output nodes was used. The input consist of the amplitude vector obtained by extraction of power spectra density amplitudes for the characteristic frequencies of the bearing used in our experimental setup, with the fault state as the output.

The four inputs in their vector order are:

Fundamental cage frequency amplitude (fcfa)

Ball passes outer raceway frequency amplitude (bpofa)

Ball spins frequency amplitude (bsfa)

Ball passes inner raceway frequency amplitude (bpifa)

The Fig. 3 shows the scheme of the system generated by ANFIS Editor GUI in Matlab. 


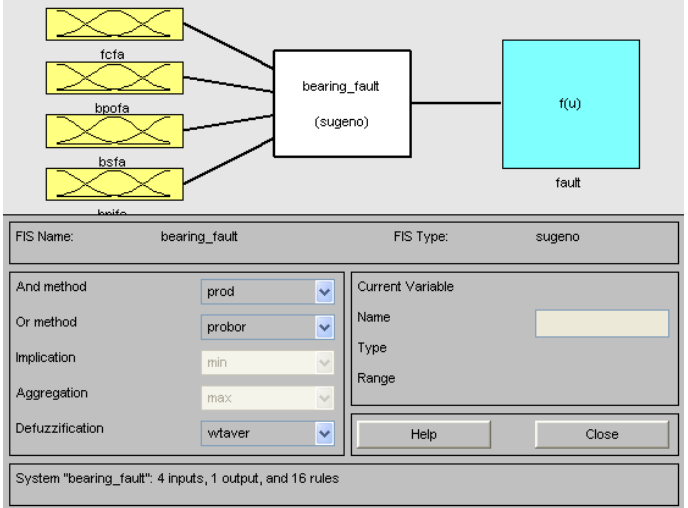

Fig. 3. Scheme of the system generated by ANFIS Editor GUI in Matlab

From our data collection we select 10 data sets each to characterize each fault state at four different speeds (namely $1500 \mathrm{rpm}, 1800 \mathrm{rpm}, 2400 \mathrm{rpm}$ and $3000 \mathrm{rpm}$ ) for three different purposes (namely, training, testing and checking of the ANFIS network), thus making a total of $10 \times 8 \times 4=320$ data sets for each purpose and a total of 960 data sets for our analysis.

Fig. 4 shows the main window with the training data sets loaded. The training data consists of 10 data sets each for each of the four speeds, making 40 data sets for normal and each of the seven fault states, thus making a total of 320 .

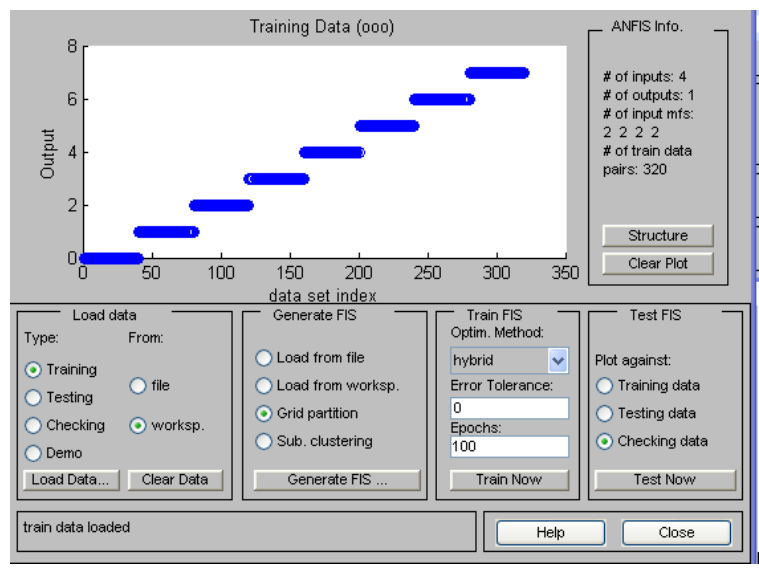

Fig. 4. The main window with the training data sets loaded

The system is loaded with the data set for training and allowed to iterate through the data set for 100 epochs. Fig. 5 shows the training error at each epoch. The error descended steeply to an acceptable level with the first few epochs and level off around the $20^{\text {th }}$ epoch. This is also attested by the command line output of the system.

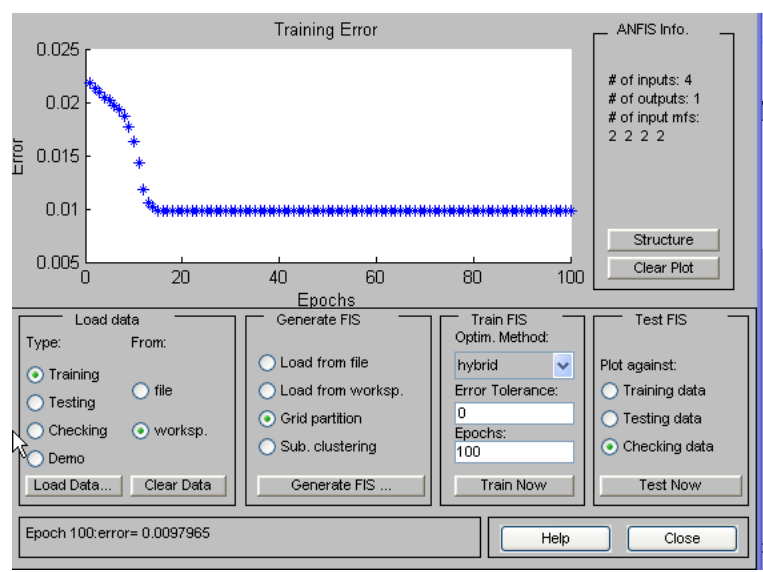

Fig. 5. The training error at each epoch
Fig. 6 shows a comparison of the error for the training and that of the error between the output generated by the trained system and that of the checking data.

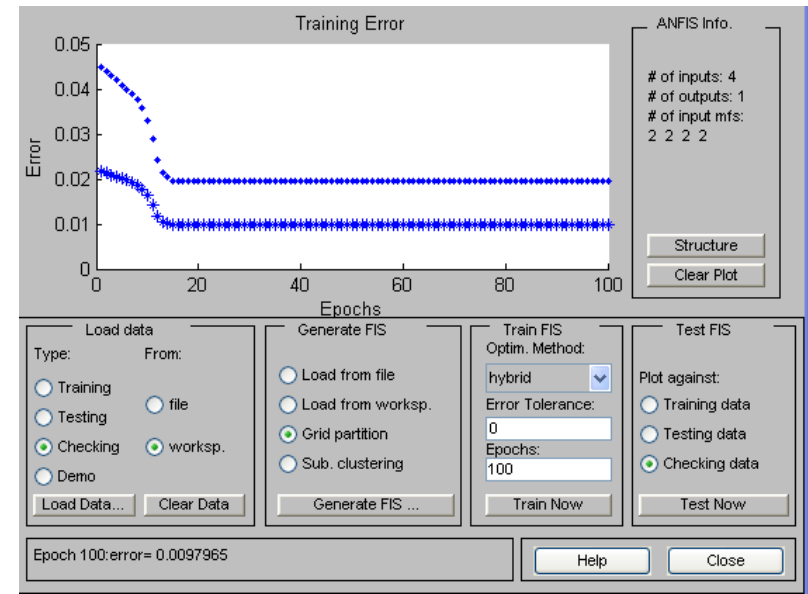

Fig. 6. Comparison of training and checking data error.

It can be seen from Fig. 7 that the structure of error level for the training and checking data sets (which are different sets of data) is very similar. This is a validation of the structural stability of the system i.e. that it will perform correctly for any set of data obtained in the same way as that of training and checking data.

ANFIS info:

Number of nodes: 55

Number of linear parameters: 80

Number of nonlinear parameters: 32

Total number of parameters: 112

Number of training data pairs: 320

Number of checking data pairs: 320

Number of fuzzy rules: 16

Start training ANFIS ...

$\begin{array}{lll}1 & 0.00979654 & 0.0193941 \\ 2 & 0.00995411 & 0.0201257\end{array}$

Designated epoch number reached --> ANFIS training completed at epoch 2 .

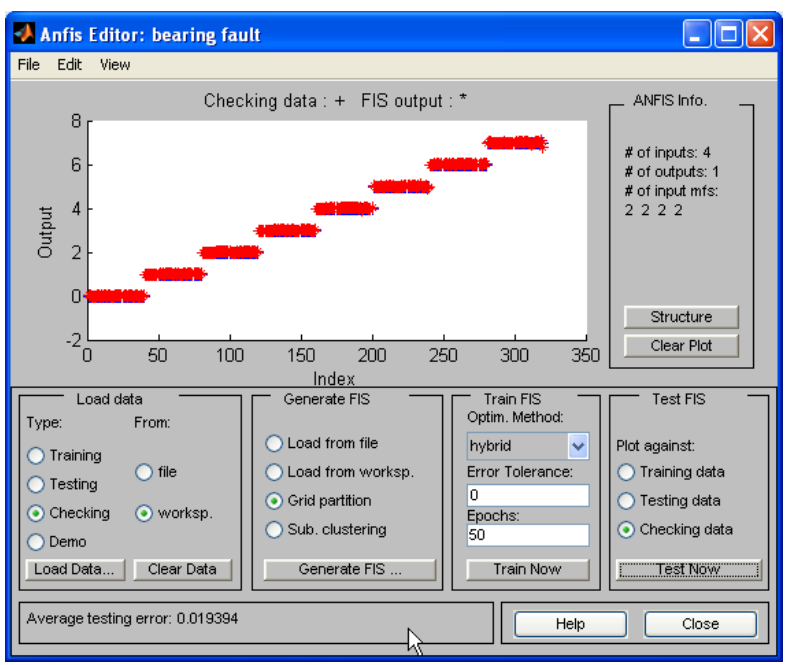

Fig. 7. Comparison of the output generated by the system with the output of checking data set

Furthermore, Fig. 7 above shows the output of the checking data in comparison to that of the output from the trained system, the two practically covers each other with little or no variation. The rule base generated by the system 
is shown diagrammatically in Fig. 8

Fig. 8 shows the rule surfaces for combinations of the inputs. Essentially rule surfaces show the output in variation to combinations of the inputs.

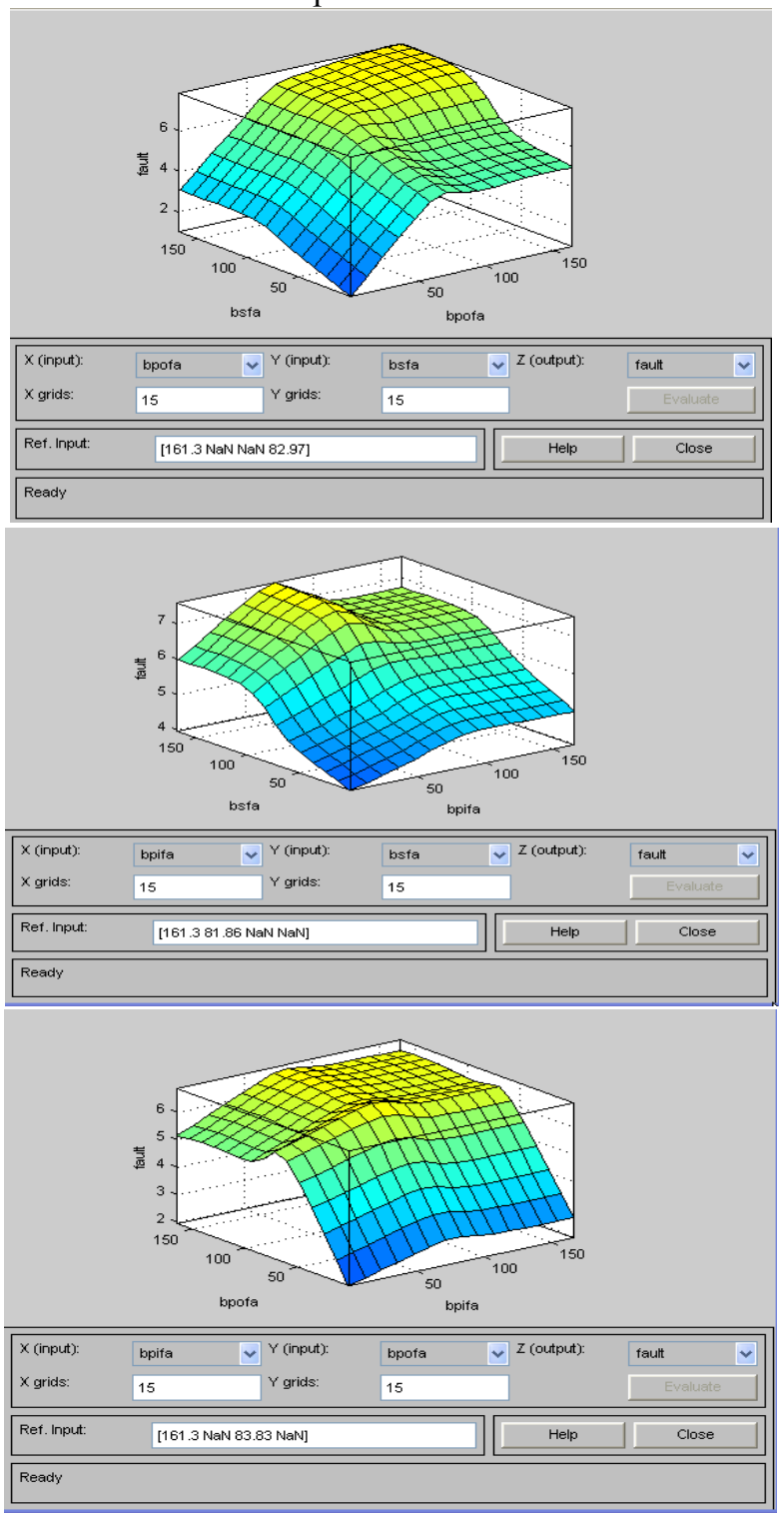

Fig. 8. The rule surfaces for combinations of the inputs.

\section{CONCLUSION}

The study thus far has shown the possibility of using vibration signal to diagnose bearing defects in rotating machinery. It has also been shown that autonomous fault diagnostic system can be developed with the use of ANFIS. Training, testing and checking results obtained for the offline implementation of the system has proven the structural stability of the system and the correctness of the approach.

\section{REFERENCES}

[1] J. T. Broch, Mechanical vibrations and shock measurements, Bruel and Kjaer, 1984, ch. 4-5.

[2] J. Morel, L. D. Revered, and P. Neau, "Vibration monitoring of heavy duty machines," in Proceedings of the 4th incipient failure detection conference, Philaadelphia, PA.1990, pp. 234-242.

[3] I. Howard, A Review of Rolling Element Bearing Vibration, Detection, Diagnosis and Prognosis, 1994.

[4] A. Tandon and A. Choudhury, "A Review of the Vibration and acoustic measurement methods for detection of defects in rolling element bearings," Tribology International, vol. 32, no. 8, pp. 469480, 1999.

[5] E. Hasbargen and Weigand, Ball and Roler Bearings - Their Theory, Design, and Application, London: K. G. Heyden \& Co. Ltd, 1958.

[6] A. Palmgren, Ball and Roller Bearing Engineering, S. H. Burbank and Co., Inc.

[7] R. B. Randall, J. Antoni, and S. Chobsaard, "The Relationship between spectral Correlation and Envelope Analysis in the Diagnostics of Bearing Faults and other Cyclostationary machines signals," Mechanical systems and signal processing, vol. 15, no. 5, pp. 945-962, 2001.

[8] M. R. Keith, Root cause failure Analysis (Plant Engineering Maintenance Series), Butterworth-Heinemann, 1994.

[9] H. Ocak and K. A. Loparo, "Estimation of the running speed and bearing defect frequencies of an induction motor from vibration data," Mechanical Systems and Signal Processing, vol. 18, pp. 515-533, 2004.

[10] T. A. Harris, Rolling Bearing Analysis, third edition, John Wiley \& Son, Inc, 1991.

[11] K. Ragulskis and A. Yurkauskas, Vibration of Bearings, 1st ed, Hemisphere Publishing Corporation, 1989.

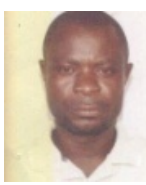

K. O. Oyedoja received is B.Eng., M. Eng., in Electrical Engineering (Computer and Communication Option) from University of Ilorin, Nigeria, Msc in Industrial and Production Engineering from University of Ibadan,

Nigeria, respectively. At present, he is with the Department of Technical Education, EACOED, Oyo, Oyo State, Nigeria and working towards his $\mathrm{PhD}$. His research interests are Artificial intelligent, Digital signal Processing, Neural Network, fuzzy logic, Matlab, etc. 